\title{
Model Reduction for Reaction-Diffusion Systems: Bifurcations in Slow Invariant Manifolds
}

\author{
Joshua D. Mengers* and Joseph M. Powers ${ }^{\dagger}$ \\ University of Notre Dame, Notre Dame, Indiana 46556, USA
}

\begin{abstract}
Slow invariant manifolds (SIM) are calculated for spatially inhomogeneous closed reactive systems to obtain a model reduction. A simple oxygen dissociation reaction-diffusion system is evaluated. SIMs are calculated using a robust method of finding the system's equilibria and integrating to find heteroclinic orbits. Diffusion effects are obtained by using a Galerkin method to project the infinite dimensional dynamical system onto a low dimensional approximate inertial manifold. This projection rigorously accounts for the coupling of reaction and diffusion processes. An analytic coupling between reaction and diffusion time scales is shown to be a function of length scale. A critical length scale is identified where reaction and diffusion time scales are equal. At this critical length scale, a supercritical pitchfork bifurcation occurs which changes the SIM.
\end{abstract}

\section{Introduction}

Accurate modeling of gas phase combustion phenomena is critical in many aerospace applications. These problems are known to display multi-scale phenomena that cause challenges in their numerical simulation known as stiffness. Verification requires grid resolution that captures the full range of scales in both space and time. Large disparity in scales induces simulations that require significant computational effort. A disparity in temporal scales can be caused by the reaction mechanism alone; the addition of diffusion introduces additional time scales. Recently, effort has been expended in identification of model reduction techniques for reactive flows in order to reduce the computational cost, while maintaining as much consistency with the underlying reactive flow physics as possible. The reviews of Griffiths ${ }^{1}$ and $\mathrm{Lu}$ and $\mathrm{Law}^{2}$ are useful. Most of the methods described therein address only reaction mechanisms. Some current research that extends these methods to systems with diffusion is detailed in Singh et al.,${ }^{3}$ Ren and Pope ${ }^{4}$ Davis,${ }^{5,6}$ Bykov and Maas, ${ }^{7}$ Lam, ${ }^{8}$ Adrover et al.,${ }^{9}$ and Goussis et al. ${ }^{10}$

The study of Davis and Skodje ${ }^{11}$ is particularly relevant. In their study, which was performed on spatially homogeneous reactive systems, the authors calculate a one-dimensional slow invariant manifold (SIM) by integrating a heteroclinic orbit between the system's physical and non-physical equilibria. This technique has recently been refined by Al-Khateeb et al. ${ }^{12}$ to examine realistic systems with a larger number of species. The SIM is a unique trajectory of the dynamical system that describes the long time dynamics of the system's evolution efficiently. The SIM has almost exclusively been used for spatially homogeneous isothermal systems.

The present work extends the SIM to reaction-diffusion systems. We first present the governing partial differential equations (PDEs). We then give reductions via a Galerkin projection method for the system of PDEs to reduce it onto an approximate inertial manifold (AIM) similar to the technique describe by Robinson. ${ }^{13}$ The result is a system of non-linear ordinary differential equations (ODEs). In the limit of small length scales, these ODEs reduce to the spatially homogeneous problem. For small, finite length scales, we find correction terms to account for diffusion. We then give results for a simple oxygen dissociation reaction with diffusion. Our analysis identifies a critical length scale at which a supercritical pitchfork bifurcation occurs. This bifurcation is shown to change the slow dynamics of the system, and therefore, the SIM.

*Ph.D. Candidate, Department of Aerospace and Mechanical Engineering, University of Notre Dame, AIAA Student Member.

${ }^{\dagger}$ Professor, Department of Aerospace and Mechanical Engineering, University of Notre Dame, AIAA Associate Fellow.

Copyright (C) 2012 by the American Institute of Aeronautics and Astronautics, Inc. The U.S. Government has a royalty-free license to exercise all rights under the copyright claimed herein for Governmental purposes. All other rights are reserved by the copyright owner. 


\section{Model}

We model a system of detailed reaction kinetics of $N$ species reacting in $J$ reactions with diffusion in one spatial dimension. The key assumptions in our model include an ideal mixture of ideal gases, negligible advection, constant volume, constant temperature, and a single constant mass diffusivity.

\section{II.A. Balance Laws}

Our governing species evolution equation is

$$
\rho \frac{\partial Y_{i}}{\partial t}+\frac{\partial j_{i}^{m}}{\partial x}=\mathrm{M}_{i} \dot{\omega}_{i}\left(Y_{n}\right), \quad \text { for } i, n \in[1, N],
$$

where for $i=1, \ldots, N, Y_{i}, j_{i}^{m}, \dot{\omega}_{i}$, and $\mathrm{M}_{i}$, are mass fraction, diffusive mass flux, molar production rate, and molecular mass of species $i$, respectively; $\rho$ is the constant density, and $T$ is the constant temperature. Our model is on a domain of $x \in[0, \ell]$ and $t \in[0, \infty)$. The initial conditions are

$$
Y_{i}(x, t=0)=\tilde{Y}_{i}(x), \quad \text { for } i \in[1, N],
$$

where the $\sim$ denotes the initial state. We select homogeneous Neumann boundary conditions:

$$
\left.\frac{\partial Y_{i}}{\partial x}\right|_{x=0}=\left.\frac{\partial Y_{i}}{\partial x}\right|_{x=\ell}=0, \quad \text { for } i \in[1, N]
$$

\section{II.B. Constitutive Equations}

To complete the system, a set of constitutive equations is specified. The mass flux is given by a simple Fick's law of diffusion,

$$
j_{i}^{m}=-\rho \mathcal{D} \frac{\partial Y_{i}}{\partial x},
$$

where $\mathcal{D}$ is the constant mass diffusivity. Thus, our homogeneous boundary conditions result in no diffusive mass flux at either boundary. The pressure, $P$, is given by the ideal gas equation of state for a mixture which obeys Dalton's law:

$$
P=\rho \bar{\Re} T\left(\sum_{i=1}^{N} \frac{\mathrm{M}_{i}}{Y_{i}}\right)^{-1},
$$

where $\overline{\mathfrak{R}}=8.314 \times 10^{7} \mathrm{erg} /(\mathrm{mol} \mathrm{K})$ is the universal gas constant. The reaction source terms are

$$
\begin{aligned}
\dot{\omega}_{i} & =\sum_{j=1}^{J} \nu_{i j} r_{j}, \quad \text { for } i \in[1, N], \\
r_{j} & =k_{j}\left(\prod_{i=1}^{N}\left(\frac{\rho Y_{i}}{\mathrm{M}_{i}}\right)^{\nu_{i j}^{\prime}}-\frac{1}{K_{j}^{c}} \prod_{i=1}^{N}\left(\frac{\rho Y_{i}}{\mathrm{M}_{i}}\right)^{\nu_{i j}^{\prime \prime}}\right), \quad \text { for } j \in[1, J], \\
k_{j} & =a_{j} T^{\beta_{j}} \exp \left(\frac{-\bar{E}_{j}}{\overline{\mathfrak{R} T}}\right), \quad \text { for } j \in[1, J], \\
K_{j}^{c} & =\left(\frac{P_{o}}{\overline{\mathfrak{R} T}}\right)^{\sum_{i=1}^{N} \nu_{i j}} \exp \left(\frac{-\sum_{i=1}^{N} \bar{g}_{i}^{o} \nu_{i j}}{\overline{\mathfrak{R} T}}\right), \quad \text { for } j \in[1, J],
\end{aligned}
$$

where for $j=1, \ldots, J, r_{j}, k_{j}$, and $K_{j}^{c}$ are the reaction rate, Arrhenius rate, and equilibrium constant of reaction $j$, respectively. The reaction-species coefficient matrix, $\nu_{i j} \equiv \nu_{i j}^{\prime \prime}-\nu_{i j}^{\prime}$, is of dimension $N \times J$ and is segregated into forward, $\nu_{i j}^{\prime}$, and reverse, $\nu_{i j}^{\prime \prime}$, reactions. Eqs. (1-9) form a complete set of equations.

\section{II.C. Model Reduction}

Here we describe a series of reductions of Eqs. (1-9). 


\section{II.C.1. Generalized Shvab-Zel'dovich}

We can further simplify the system using reductions similar to those described by Lam and Bellan, ${ }^{14}$ who label them as generalized Shvab-Zel'dovich relations. We construct a matrix $\varphi_{l i}$ of dimension $L \times N$, where the $L$ row vectors span the left null space of matrix $\nu_{i j}$ :

$$
\sum_{i=1}^{N} \varphi_{l i} \nu_{i j}=0, \quad \text { for } l \in[1, L], \text { and } j \in[1, J]
$$

A good choice for $\varphi_{l i}$ is the stoichiometric coefficient matrix because the stoichiometric constraints reflect the linear dependencies on the system. Operating on Eq. (6) with $\varphi_{l i}$ and summing on $i$, we get

$$
\sum_{i=1}^{N} \varphi_{l i} \sum_{j=1}^{J} \nu_{i j} r_{j}=\sum_{j=1}^{J} r_{j} \underbrace{\sum_{i=1}^{N} \varphi_{l i} \nu_{i j}}_{=0}, \quad \text { for } l \in[1, L],
$$

therefore

$$
\sum_{i=1}^{N} \varphi_{l i} \dot{\omega}_{i}=0, \quad \text { for } l \in[1, L] .
$$

With this simplification, we can apply the operator $\varphi_{l i}$ on Eq. (1), use the diffusive mass flux from Eq. (4), and sum over all species to obtain

$$
\frac{\partial}{\partial t}\left(\sum_{i=1}^{N} \varphi_{l i} \frac{Y_{i}}{\mathrm{M}_{i}}\right)-\mathcal{D} \frac{\partial^{2}}{\partial x^{2}}\left(\sum_{i=1}^{N} \varphi_{l i} \frac{Y_{i}}{\mathrm{M}_{i}}\right)=0, \quad \text { for } l \in[1, L] .
$$

If the initial conditions are spatially homogeneous in the quantity

$$
\sum_{i=1}^{N} \varphi_{l i} \frac{Y_{i}}{\mathrm{M}_{i}}, \quad \text { for } l \in[1, L],
$$

and there are no perturbations from the boundary conditions, then Eq. (13) can be integrated to yield

$$
\sum_{i=1}^{N} \varphi_{l i} \frac{Y_{i}}{\mathrm{M}_{i}}=\sum_{i=1}^{N} \varphi_{l i} \frac{\tilde{Y}_{i}}{\mathrm{M}_{i}}, \quad \text { for } l \in[1, L]
$$

which is a set of $L$ algebraic relations for $Y_{i}$. This suggests that we need not solve for the nonlinear dynamics of all $N$ species, but can focus attention on $N-L$ species and use Eq. (15) to determine the dynamics of the remaining ones. Note that each individual $Y_{i}(x)$ need not be spatially homogeneous, only the values of the constraints defined in Eq. (14). Each row vector of $\varphi_{l i}$ can be correlated to a physical constraint on our system (i.e. the conservation of elements).

\section{II.C.2. Transformation to Reduced Variables}

Let us now transform the mass fractions into specific mole concentrations, $\hat{z}_{i}$, where

$$
\hat{z}_{i} \equiv \frac{Y_{i}}{\mathrm{M}_{i}}, \quad \text { for } i \in[1, N]
$$

We can use the constraints in Eq. (15) to obtain a set of reduced variables. We choose the first $N-L$ species as our reduced variables,

$$
z_{i}=\hat{z}_{i}, \quad \text { for } i \in[1, N-L]
$$

We then couple the values of $\hat{z}_{i}$ for $i \in[1, N]$ to the values of $z_{i}$ by the linear relation

$$
\hat{z}_{i}(x, t)=\check{z}_{i}+\hat{\nu}_{i n} z_{n}(x, t), \quad \text { for } i \in[1, N] \text { and } n \in[1, N-L],
$$


where $\check{z}_{i}$ are the values of specific mole concentration when $z_{n}=0$ for all $n \in[1, N-L]$, and $\hat{\nu}_{i n}$ is a coefficient matrix of dimension $N \times(N-L)$ that couples the variations in $z_{n}$ to $\hat{z}_{i}$. We define $\hat{\nu}_{i n}$ to be a full rank matrix that spans the column space of $\nu_{i j}$, where $\nu_{i j}$ need not be full rank. Therefore, $\sum_{i=1}^{N} \varphi_{l i} \hat{\nu}_{i n}=0$ for $l \in[1, L]$, and $n \in[1, N-L]$. This reduction is not unique, and the following is a technique to obtain $\hat{\nu}_{i n}$ : reduce the transpose of the $\nu_{i j}$ matrix to a row-echelon form, truncate the final $J-(N-L)$ rows of zeros in this echelon form, and take the transpose of this truncation to form $\hat{\nu}_{i n}$. In this construction of $\hat{\nu}_{i n}$, the first $N-L$ rows form a sub-matrix which is an $(N-L) \times(N-L)$ identity matrix, indicating that the reduced variables are the first $N-L$ species.

Operating $\varphi_{l i}$ on Eq. (1), substituting Eq. (16) for mass fraction, and summing on $i$ species, yields the same $L$ constraints given in Eq. (15). The remaining $N-L$ ODEs are the governing equations in the reduced variables, which are

$$
\frac{\partial z_{i}}{\partial t}=\frac{\dot{\omega}_{i}\left(z_{n}\right)}{\rho}+\mathcal{D} \frac{\partial^{2} z_{i}}{\partial x^{2}}, \quad \text { for } i, n \in[1, N-L],
$$

where $\dot{\omega}_{i}$ is now only a function of the reduced variables. For this system we admit spatial variations in each reduced variable; however the species constraints given in Eq. (15) remain spatially homogeneous.

\section{II.C.3. Galerkin Reduction to ODEs}

To analyze Eq. (19), we apply a Galerkin projection onto an AIM. ${ }^{13}$ To accomplish this, we assume a spectral decomposition of

$$
z_{i}(x, t)=\sum_{m=0}^{\infty} z_{i, m}(t) \phi_{m}(x), \quad \text { for } i \in[1, N-L]
$$

where $z_{i, m}(t)$ is the $m^{t h}$ time-dependent amplitude associated with species $i$, and $\phi_{m}(x)$ are corresponding basis functions which we select. This projection is only in the reduced variables; our constraints, which must be constant in space, govern the evolution of the remaining species. We choose $\phi_{m}(x)$ as the eigenfunctions of the diffusion operator that match the boundary conditions. This reduces to the eigenvalue problem, $\partial^{2} / \partial x^{2} \phi_{m}=-\mu_{m}^{2} \phi_{m}$, whose solution is the complete basis

$$
\phi_{m}=\cos \left(\frac{m \pi x}{\ell}\right), \quad \text { for } m \in[0, \infty)
$$

These basis functions are orthogonal, $\left\langle\phi_{m}, \phi_{n}\right\rangle=0, n \neq m$, and their eigenvalues are real, given by $\mu_{m}=m \pi / \ell$. By substituting Eq. (20) into Eq. (19), taking the inner product with each basis function, $\phi_{n}$, and taking advantage of the orthogonality of the basis functions, we obtain an infinite system of ODEs for the evolution of the amplitudes:

$$
\frac{d z_{i, m}}{d t}=\frac{<\phi_{m}, \dot{\omega}_{i}\left(\sum_{n=0}^{\infty} z_{i, n} \phi_{n}\right) / \rho>}{<\phi_{m}, \phi_{m}>}-\mathcal{D} \mu_{m}^{2} z_{i, m}, \quad \text { for } i \in[1, N-L], \text { and } m \in[0, \infty),
$$

where the initial conditions of the amplitudes are given by

$$
\tilde{z}_{i, m}=\frac{<\phi_{m}, \tilde{Y}_{i} / \mathrm{M}_{i}>}{<\phi_{m}, \phi_{m}>}, \quad \text { for } i \in[1, N-L], \text { and } m \in[0, \infty)
$$


For our choice of basis functions, $<\phi_{0}, \phi_{0}>=\ell$ and $<\phi_{m}, \phi_{m}>=\ell / 2$ for $m \geq 1$. This leaves the system of ODEs as

$$
\begin{gathered}
\frac{d z_{i, 0}}{d t}=\underbrace{\frac{1}{\ell} \int_{0}^{\ell} \frac{\dot{\omega}_{i}\left(z_{n}(x, t)\right)}{\rho} d x}_{\equiv \dot{\omega}_{i, 0}}, \quad \text { for } i, n \in[1, N-L], \\
z_{i, 0}(0)=\tilde{z}_{i, 0}=\underbrace{\frac{1}{\ell \mathrm{M}_{i}} \int_{0}^{\ell} \tilde{Y}_{i}(x) d x, \quad \text { for } i \in[1, N-L],}_{\equiv \dot{\omega}_{i, m}} \\
\frac{d z_{i, m}}{d t}=\underbrace{\frac{2}{\ell} \int_{0}^{\ell} \frac{\dot{\dot{\omega}\left(z_{n}(x, t)\right)}}{\rho} \cos \left(\frac{m \pi x}{\ell}\right) d x}_{z_{i, m}(0)=\tilde{z}_{i, m}=}-\frac{\pi^{2} m^{2} \mathcal{D}}{\ell^{2}} z_{i, m}, \\
\text { for } i, n \in[1, N-L], \text { and } m \in[1, \infty),
\end{gathered}
$$

For small $\ell$ and $m \geq 1$, the diffusion term dominates the reaction term, which makes the $m \geq 1$ amplitudes decay rapidly. The integration of the $\dot{\omega}_{i, m}$ terms from Eqs. (24) and (26) can be performed analytically using trigonometric identities since our molar production rates are polynomial and our basis functions are cosines.

Truncating at a finite $M$ reduces the infinite system of ODEs in Eqs. (24) and (26) to the system of $(M+1)(N-L)$ ODEs,

$$
\begin{array}{rlrl}
\frac{d z_{i, m}}{d t}=\dot{\omega}_{i, m}-\frac{m^{2} \pi^{2} \mathcal{D}}{\ell^{2}} z_{i, m}, & & \text { for } i \in[1, N-L], \text { and } m \in[0, M], \\
z_{i, m}(0)=\tilde{z}_{i, m}, & \text { for } i \in[1, N-L], \text { and } m \in[0, M] .
\end{array}
$$

For very small $\ell$, a truncation at $M=0$ is appropriate in which case Eqs. (28) and (29) reduce to a spatially homogeneous system,

$$
\begin{aligned}
\frac{d z_{i}}{d t} & =\dot{\omega}_{i}\left(z_{n}, T\right), & & \text { for } i, n \in[1, N-L], \\
z_{i}(0) & =\tilde{z}_{i}, & & \text { for } i, n \in[1, N-L] .
\end{aligned}
$$

For this case $\pi^{2} \mathcal{D} / \ell^{2}$ is large, meaning that the diffusion term will dominate the reaction term for any $m>0$. This will make the diffusion much faster than reaction, all spatial inhomogeneities equilibrate quickly, and a spatially homogeneous system will be recovered.

\section{Oxygen Dissociation Reaction Mechanism}

We evaluate the oxygen dissociation reaction mechanism,

$$
\mathrm{O}_{2}+\mathrm{M} \leftrightharpoons \mathrm{O}+\mathrm{O}+\mathrm{M}
$$

which is extracted from Miller et al., ${ }^{15}$ whose Arrhenius rate constants are $a=1.85 \times 10^{11} \mathrm{~cm}^{3} /(\mathrm{mol} \mathrm{s} \sqrt{K})$, $\beta=0.5$, and $\bar{E}=4.0009 \times 10^{12} \mathrm{erg} / \mathrm{mol}$. This system has $N=2$ species and $J=1$ reactions. Conservation of elements yields $L=1$ algebraic constraint that allows the system to be reduced to $N-L=1$ reduced variable; we choose to evaluate the specific moles of $O$ as $z$; the evolution of $O_{2}$, identified as $\hat{z}_{2}$, is coupled to the evolution of $z$ by the algebraic constraint. Note here that we have dropped the $i$ subscript in our $z$ notation for simplicity. This leaves us with the reaction-species coefficient, species constraint, and species reduction matrices as

$$
\nu_{i j}=\left[\begin{array}{c}
2 \\
-1
\end{array}\right], \quad \varphi_{l i}=\left[\begin{array}{ll}
1 & 2
\end{array}\right], \quad \hat{\nu}_{i j}=\left[\begin{array}{c}
1 \\
-\frac{1}{2}
\end{array}\right],
$$

respectively. For this system $\nu_{i j}$, dimension $N \times J$, happens to be full rank and therefore the same dimension as $\hat{\nu}_{i j}$, dimension $N \times(N-L)$; this is not generally the case. The system is taken to be isochoric at 
$\rho=1.6 \times 10^{-4} \mathrm{~g} / \mathrm{cm}^{3}$ and isothermal at $T=5000 \mathrm{~K}$. The species' Gibbs free energy is obtained directly from the polynomial fit in the CHEMKIN thermodynamics database ${ }^{16}$ which yields $\bar{g}_{O}^{o}=-7.53951 \times 10^{12} \mathrm{erg} / \mathrm{mol}$, and $\bar{g}_{\mathrm{O}_{2}}^{o}=-1.34636 \times 10^{13} \mathrm{erg} / \mathrm{mol}$. The constraints form the following algebraic relationships for the full species concentrations as functions of reduced species

$$
\left[\begin{array}{l}
\hat{z}_{1}(x, t) \\
\hat{z}_{2}(x, t)
\end{array}\right]=\left[\begin{array}{c}
0 \\
3.125 \times 10^{-2}
\end{array}\right] \mathrm{mol} / \mathrm{g}+\left[\begin{array}{r}
1 \\
-\frac{1}{2}
\end{array}\right] z(x, t) .
$$

\section{Results and Discussion}

\section{IV.A. Spatially Homogeneous}

We start by examining the spatially homogeneous $(M=0)$ system, which is governed by the equation

$$
\frac{d z}{d t}=\left(249.841 \frac{g}{m o l s}\right)-\left(74734.8 \frac{m o l}{g s}\right) z^{2}-\left(172406 \frac{m o l^{2}}{g^{2} s}\right) z^{3} .
$$

The SIM analysis is trivial because the spatially homogeneous system is one-dimensional; nevertheless, we proceed to find the degenerate one-dimensional SIM for this system as it proves useful to the spatially inhomogeneous analysis. To identify the branches of the SIM, we first must find the system's equilibria via an algebraic method and then characterize these equilibria as sources or sinks using a local linear analysis. We identify three finite equilibria, listed here with the eigenvalue of their Jacobian, which is the slope for our one-dimensional example,

$$
\begin{array}{ll}
z_{R 1}=0.054495 \mathrm{~mol} / \mathrm{g}, & \lambda_{R 1}=-9681.33 \mathrm{~s}^{-1}, \\
z_{R 2}=-0.0625 \mathrm{~mol} / \mathrm{g}, & \lambda_{R 2}=7321.46 \mathrm{~s}^{-1}, \\
z_{R 3}=-0.425475 \mathrm{~mol} / \mathrm{g}, & \lambda_{R 3}=-30036.2 \mathrm{~s}^{-1} .
\end{array}
$$

Here we note that only a portion of the domain, $z \in[0 \mathrm{~mol} / \mathrm{g}, 0.0625 \mathrm{~mol} / \mathrm{g}]$, is physically realizable. The equilibrium $R_{1}$ is the physical equilibrium sink, while the nonphysical $R_{2}$ and $R_{3}$ are a source and a sink, respectively. The SIM is calculated by integrating a heteroclinic orbit between a nonphysical equilibrium with one positive eigenvalue and the physical sink equilibrium. One branch of the SIM is the portion of the domain between $R_{2}$ and $R_{1}$, while the other branch is located between $R_{1}$ and an equilibrium source located at $z=+\infty ; ;^{11,12}$ the remainder of our analysis will focus on the former branch. A plot of $\dot{\omega}(z)$ in Fig. 1 shows these equilibria and their slopes (eigenvalues of their Jacobian) as well as the branch of the SIM, shown in red.

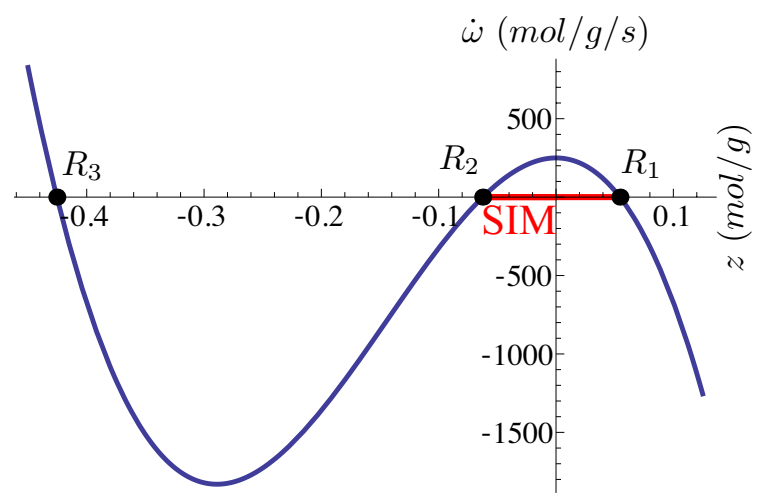

Figure 1. Spatially homogeneous molar production rate as a function of specific moles.

\section{IV.B. Diffusion Correction}

We next examine a spatially inhomogeneous system to evaluate changes in the SIM for systems with small length scale, therefore fast diffusion time scale. Because our length scale is small, we can maintain full resolution of the spatio-temporal dynamics and still truncate at $M=1$. Under this truncation, the evolution 
equations, Eq. (28), for the oxygen dissociation system become

$$
\begin{aligned}
\frac{d z_{0}}{d t} & =\left(249.841 \frac{g}{m o l s}\right)-\left(74734.8 \frac{m o l}{g s}\right)\left(z_{0}^{2}+\frac{z_{1}^{2}}{2}\right)-\left(172406 \frac{m o l^{2}}{g^{2} s}\right)\left(z_{0}^{3}+\frac{3 z_{0} z_{1}^{2}}{2}\right) \\
\frac{d z_{1}}{d t} & =-\left(74734.8 \frac{\mathrm{mol}}{g s}\right) 2 z_{0} z_{1}-\left(172406 \frac{m o l^{2}}{g^{2} s}\right)\left(3 z_{0}^{2} z_{1}+\frac{3 z_{1}^{3}}{4}\right)-\frac{\pi^{2} \mathcal{D}}{\ell^{2}} z_{1}
\end{aligned}
$$

where the diffusion coefficient $\mathcal{D}=8.1 \mathrm{~cm}^{2} / \mathrm{s}$ is approximated from an average of the ordinary multicomponent diffusion coefficients in the CHEMKIN TRANSPORT database, ${ }^{17}$ and the length scale, $\ell$, is left as a free parameter at this stage.

We note that the time evolution of the $z_{0}$ term, Eq. (39), remains identical to the spatially homogeneous evolution when $z_{1}=0$, and the time evolution of the $z_{1}$ term, Eq. (40), is zero when $z_{1}=0$. This means that the entire spatially homogeneous phase space, including the spatially homogeneous equilibria, remains as a subspace in the diffusion-modified system's phase space. We note here that because of a physical symmetry in the system $(\cos (\pi x / \ell)$ behaves the same as $-\cos (\pi x / \ell))$ the dynamics of $-z_{1}$ are the mirror image of $z_{1}$.

The eigenvalues of the Jacobian linearized about these spatially homogeneous equilibria are modified in the reaction-diffusion system such that they can be described analytically. The original spatially homogeneous eigenvalue is retained and an additional diffusion-modified eigenvalue is given by

$$
\lambda_{1}=\lambda_{0}-\frac{\pi^{2} \mathcal{D}}{\ell^{2}}
$$

where $\lambda_{0}$ is the eigenvalue of the spatially homogeneous equilibria. This means that the additional diffusionmodified modes at the spatially homogeneous equilibria are always more negative, or more stable.

We now define local time scales as the reciprocal of the eigenvalues,

$$
\tau_{m}=\frac{1}{\lambda_{m}}, \quad \text { for } m \in[0,1]
$$

and a diffusion time scale is given by

$$
\tau_{\mathcal{D}}=\frac{\ell^{2}}{\pi^{2} \mathcal{D}} .
$$

The spatially homogeneous time scale and eigenvalue, $\tau_{0}$ and $\lambda_{0}$, correlate to the dynamics of reaction only, while the diffusion-modified time scale and eigenvalue, $\tau_{1}$ and $\lambda_{1}$, correlate to the coupled reaction-diffusion dynamics.

In Fig. 2, we see the time scale for the spatially homogeneous equilibria $R_{1}$ and $R_{2}$ as a function of $\ell$. We see that the reaction-only time scales (solid lines) remain constant, independent of $\ell$, while the diffusion-

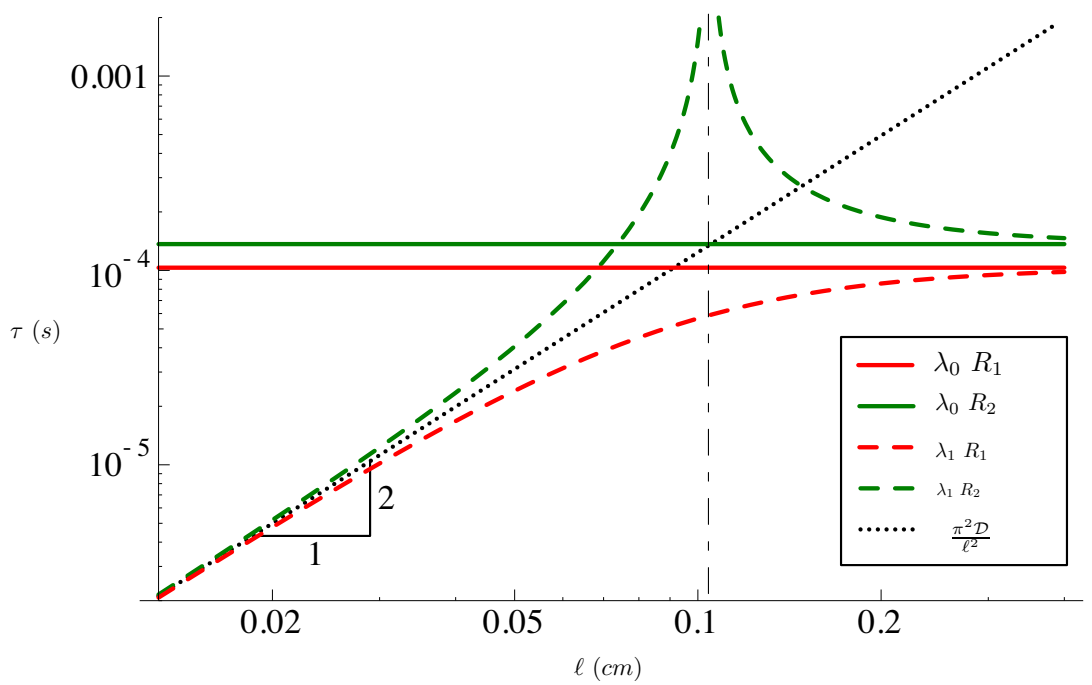

Figure 2. Local time scales in the neighborhood of the equilibria. 
modified time scales (dashed lines) approach the reaction time scale for long length scales and the diffusion time scale (dotted line) for short length scales. There is a transition region over the length scales where the reaction and diffusion time scales are approximately the same value, which occurs for $\ell \sim \sqrt{\mathcal{D} \tau_{0}}$. For $R_{1}$, since the reaction eigenvalue is negative, the diffusion-modified time scale gradually shifts between reaction and diffusion; however, for $R_{2}$ there is a sign change in the diffusion-modified time scale. This sign change occurs at a critical length scale $\ell=\ell_{c}=0.104495 \mathrm{~cm}$, where a supercritical pitchfork bifurcation of equilibrium $R_{2}$ occurs. This is shown in Fig. 3 where the solid lines indicate branches with saddle characters, and the

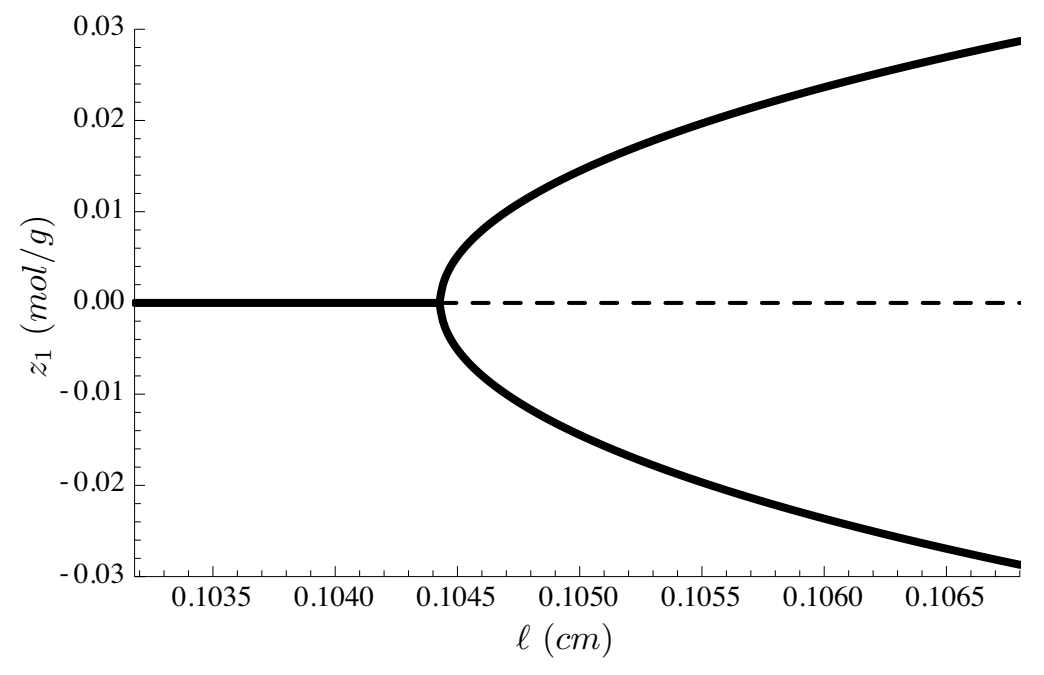

Figure 3. Locus of equilibria near $R_{2}$.

dashed line indicates a branch with source character. We name the new equilibrium from this bifurcation $R_{2}^{\prime}$ This bifurcation affects the slow dynamics of the system. For $\ell<\ell_{c}$ the SIM is the spatially homogeneous axis from $R_{2}$ to $R_{3}$; for $\ell>\ell_{c}$ the SIM is the heteroclinic orbit from the unstable eigenvector of $R_{2}^{\prime}$ to $R_{3}$.

We now choose to evaluate this system for three length scales: $\ell=\{0.0334,0.105,0.334\} \mathrm{cm}$. To better visualize the changes in the dynamics of the entire system, we transform into Poincaré sphere, by the change of coordinates

$$
\begin{aligned}
& \eta_{0}=\frac{z_{0}}{\sqrt{\alpha+z_{0}^{2}+z_{1}^{2}}} \\
& \eta_{1}=\frac{z_{1}}{\sqrt{\alpha+z_{0}^{2}+z_{1}^{2}}}
\end{aligned}
$$

where $\alpha$ is a scaling parameter to facilitate visualization. This coordinate transform has two inverse transformations; we choose the transformation with positive roots,

$$
\begin{aligned}
z_{0} & =\frac{\alpha \eta_{0}}{\sqrt{1-\eta_{0}^{2}-\eta_{1}^{2}}}, \\
z_{1} & =\frac{\alpha \eta_{1}}{\sqrt{1-\eta_{0}^{2}-\eta_{1}^{2}}} .
\end{aligned}
$$

Infinity in the $z$ coordinates transforms to the unit circle in the $\eta$ coordinates. The evolution equations, Eqs. (39) and (40), transform to

$$
\begin{aligned}
\frac{d \eta_{0}}{d t} & =\frac{\partial \eta_{0}}{\partial z_{0}} \frac{d z_{0}}{d t}+\frac{\partial \eta_{0}}{\partial z_{1}} \frac{d z_{1}}{d t} \\
\frac{d \eta_{1}}{d t} & =\frac{\partial \eta_{1}}{\partial z_{0}} \frac{d z_{0}}{d t}+\frac{\partial \eta_{1}}{\partial z_{1}} \frac{d z_{1}}{d t}
\end{aligned}
$$

Because the dynamics of the system are symmetric about the $z_{0}$ axis, they are subsequently symmetric about the $\eta_{0}$ axis. For this reason we only display $\eta_{1} \geq 0$. 
We see in Fig. 4 the Poincaré phase space $(\alpha=0.0625)$ for the three length scales. In this figure the black dots are equilibria, the gray triangle is the physical domain (where the concentrations of both species are positive) and the hashed region in the left of the of the plots are outside of the physical equilibrium's basin of attraction. In Fig. 4a we see the dynamics for $\ell=0.0334 \mathrm{~cm}<\ell_{c}$; here we see the green dashed trajectories
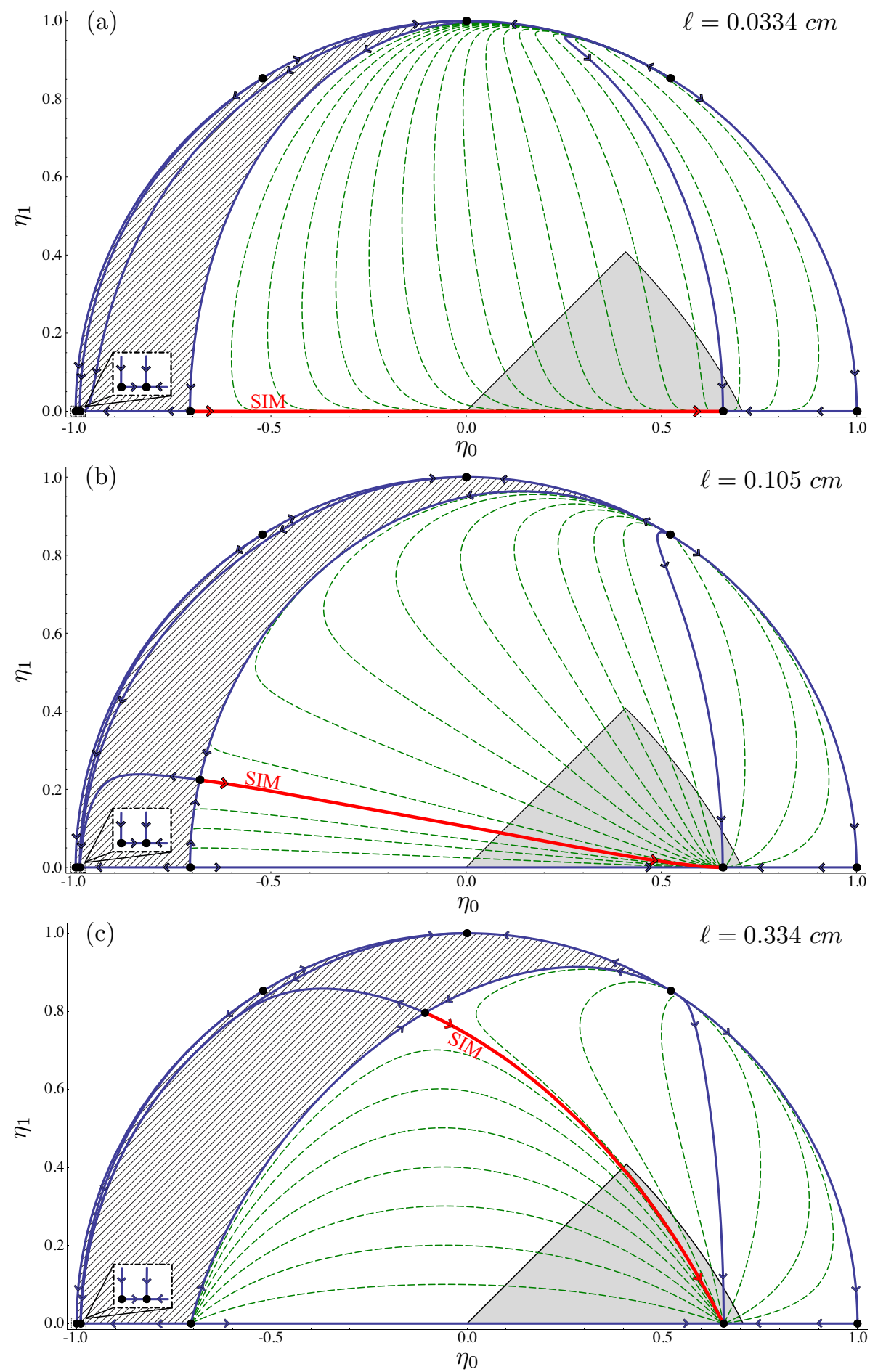

Figure 4. Poincaré sphere map for positive $\eta_{1}$ at various length scales.

collapse to the red SIM, which is the spatially homogeneous axis. This displays the fact that the diffusion time scale for this length is faster than the reaction time scale; therefore, the spatial inhomogeneities decay rapidly due to diffusion, and the reaction governs the slow dynamics. In Fig. $4 \mathrm{~b}$ we see the dynamics for 
$\ell=0.105 \mathrm{~cm}$, which is marginally larger than $\ell_{c}$. For this length we see the effects of the bifurcation, and now the SIM emanates from the unstable eigenvector of $R_{2}^{\prime}$. The green trajectories now do not collapse to the spatially homogeneous axis, instead the similar reaction and diffusion time scales bring the trajectories to the equilibrium with $\eta_{0}$ and $\eta_{1}$ decaying at approximately equal rates. We also note that in Fig. 4b the trajectories do not collapse to the SIM as they did in Fig. 4a. In Fig. 4c we see the dynamics for $\ell=0.334 \mathrm{~cm}>\ell_{c}$; here the SIM still emanates from the unstable eigenvector of $R_{2}^{\prime}$ and the bifurcation has progressed further from the spatially homogeneous axis. The green trajectories now actually diverge from the spatially homogeneous axis. This is caused by the nonlinear effects of fast reaction; the slower diffusion doesn't play a large roll in the evolution of this system. We note that in Fig. 4c the trajectories again collapse onto the SIM more than they did in Fig. 4b, but still not as much as they did in Fig. 4a.

\section{Conclusions}

Our evaluation of the dynamics of reaction-diffusion systems has identified an analytic scaling between reaction and diffusion time scales that is a function of length scale. This scaling identified a critical length scale, where the reaction and diffusion time scales are equal. The slow dynamics of the system for length scales smaller than the critical length are governed by spatially homogeneous reaction dynamics. At the critical length scale a supercritical pitchfork bifurcation occurs. For length scales longer than the critical length, this bifurcation changes the slow dynamics of the system, and a new SIM is identified that emanates from the unstable eigenvector of the bifurcated equilibrium. Finally, the present results, though limited to highly restricted reaction-diffusion models, demonstrate some of the daunting challenges facing the combustion modeling community who wish to use models which have been rationally reduced. At this point, it is likely that insights can be gained for low-dimensional systems; extension to higher dimension systems, be it via addition of more detailed reactions or more spatially inhomogeneous modes, will rapidly overwhelm most present analysis strategies.

\section{Acknowledgments}

The authors recognize the support of the National Science Foundation (NSF) under Grant No. CBET0650843. J. D. Mengers also recognizes the support from the University of Notre Dame Department of Applied and Computational Mathematics and Statistics fellowship.

\section{References}

\footnotetext{
${ }^{1}$ Griffith, J. F., "Reduced kinetic-models and their application to practical combustion systems," Progress in Energy and Combustion Science, Vol. 21, No. 1, 1995, pp. 25-107.

${ }^{2} \mathrm{Lu}, \mathrm{T} . \mathrm{F}$. and Law, C. K., "Toward accommodating realistic fuel chemistry in large-scale computations," Progress in Energy and Combustion Science, Vol. 35, No. 2, 2009, pp. 192-215.

${ }^{3}$ Singh, S., Powers, J. M., and Paolucci, S., "On slow manifolds of chemically reactive systems," Journal of Chemical Physics, Vol. 117, No. 4, 2002, pp. 1482-1496.

${ }^{4}$ Ren Z. and Pope, S. B., "Transport-chemistry coupling in the reduced description of reactive flows," Combustion Theory and Modeling, Vol. 11, No. 5, 2007, pp. 715-739.

${ }^{5}$ Davis, M. J., "Low-dimensional manifolds in reaction-diffusion equations. 1. Fundamental aspects," Journal of Physical Chemistry A, Vol. 110, No. 16, 2006, pp. 5235-5256.

${ }^{6}$ Davis, M. J., "Low-dimensional manifolds in reaction-diffusion equations. 2. Numerical analysis and method development," Journal of Physical Chemistry A, Vol. 110, No. 16, 2006, pp. 5257-5272.

${ }^{7}$ Bykov, V. and Maas, U., "The extension of the ILDM concept to reaction-diffusion models," Combustion Theory and Modeling, Vol. 11, No. 6, 2007, pp. 839-862.

${ }^{8}$ Lam, S. H., "Reduced chemistry-diffusion coupling," Combustion Science and Technology, Vol. 179, No. 4, 2007, pp. 767786.

${ }^{9}$ Adrover, A., Creta, F., Giona, M., and Valorani, M., "Stretching-based diagnostics and reduction of chemical kinetic models with diffusion," Journal of Computational Physics, Vol. 225, No. 2, 2007, pp. 1442-1471.

${ }^{10}$ Goussis, D. A., Valorani M., Creta, F., and Najm, H. N., "Reactive and reactive-diffusive time scales in stiff reactiondiffusion systems," Progress in Computational Fluid Dynamics, Vol. 5, No. 6, 2005, pp. 316-326.

${ }^{11}$ Davis, M. J. and Skodje, R. T., "Geometric investigation of low-dimensional manifolds in systems approaching equilibrium," Journal of Chemical Physics, Vol. 111, No. 3, 1999, pp. 859-874.

${ }^{12}$ Al-Khateeb, A. N., Powers, J. M., Paolucci, S., Sommese, A. J., Diller, J. A., Hauenstein, J. D., and Mengers, J. D., "One-dimensional manifolds for spatially homogeneous systems," Journal of Chemical Physics, Vol. 131, No. 2, 2009 , pp. 024118.
} 
${ }^{13}$ Robinson, J., Infinite-Dimensional Dynamical Systems: From Basic Concepts to Actual Calculations, Cambridge University Press, Cambridge, U.K., 2001, pp. 197-198, 371.

${ }^{14}$ Lam, S. H. and Bellan, J., "On de-coupling of Shvab-Zel'dovich variables in the presence of diffusion," Combustion and Flame, Vol. 132, No. 4, 2003, pp. 691-696.

${ }^{15}$ Miller, J. A., Mitchell, R. E., Smooke, M. D., and Kee, R. J., "Toward a Comprehensive Chemical Kinetic Mechanism for the Oxidation of Acetylene: Comparison of Model Predictions with Results from Flame and Shock Tube Experiments," Proceedings of the Nineteenth Symposium (International) on Combustion, Combustion Inst., Pittsburgh, PA, 1982 , pp. 181196.

${ }^{16}$ Kee, R. J., Rupley, F. M., and Miller, J. A., Sandia National Laboratories Report No. SAND878215B, 1992.

${ }^{17}$ Kee, R. J., Dixon, L. G., Warnatz, J., Coltrin, M. E., and Miller, J. A., "A Fortran computer code package for the evaluation of gas-phase multicomponent transport properties," Report No. SAND86-8246, Sandia National Laboratories, 1991. 